the cortex against electrocorticographic data. Histologic analysis revealed the presence of HAI in the posterio-medial portion of the resected cortex, which corresponded to the site of seizure generalization. HAI were present at the resection margin. Immunohistochemistry was largely non-contributory. HAI is a rare but emerging entity that is associated with epilepsy. To our knowledge, the distribution of inclusions in HAI has never been mapped to electrophysiologic data. In our case, seizure generalization correlated with the inclusions distribution. This suggests that the inclusions may: 1) play a role in epileptogenesis; or, 2) be a biomarker of disease distribution. Finally, the presence of the HAI at the resection margin may foreshadow future seizure activity in this patient.

\section{White Matter Changes In Cardiac Arrest Encephalopathy}

\section{Alturkustani ${ }^{1,2}$, L-C. Ang $^{1}$}

${ }^{1}$ Division of Neuropathology, London Health Sciences Centre; University of Western, London, Canada; ${ }^{2}$ Department of Pathology, King Abdulaziz University and Hospital, Jeddah, Saudi Arabia

$$
\text { doi:10.1017/cjn.2015.259 }
$$

Background: White matter changes are common finding during brain autopsies especially in elderly. Although there are many studies applying radiological-pathological correlation on these lesions, their pathogenesis is still unclear. However, a number of possible causes have been suggested including: hypoxic-ischemia, altered blood brain barrier permeability, vascular pathology and chronic hypoperfusion. As usually there is a multiplicity of causes in any individual case, it is very difficult to pinpoint the major causal factor contributing to observed pathological changes. In this study, we document the white matter pathology in global acute hypoxic/ischemic injury due to cardiac arrest as the major causal factor.

Method: We retrieve 16 cases of cardiac arrest encephalopathy in our archive with post arrest survival range from 6 hours to 14 days. Several special, and immunohistochemical stains were used to study the axonal and myelin pathology.

Result: The pathogenicity of the cardiac arrest was confirmed in all cases by demonstrating the expected gray matter pathology, albeit in varying degree of severity. The white matter changes range from unremarkable in the first 2 days, evidence of cerebral edema (visualized from $3^{\text {rd }}$ day on), and early axonal degeneration, to diffuse myelin pallor secondary to marked axonal loss on day 14 .

Conclusion: The white matter changes in post cardiac arrest are mainly due to early cerebral edema and axonal degeneration and the effect on myelin is secondary.

\section{Role of Chitinase 3-like-1 in CNS inflammation}

\section{C.A. Wiley}

University of Pittsburgh, Pittsburgh, Pennsylvania, USA

doi:10.1017/cjn.2015.260
The Innate Immune Response (IIR) is an evolutionarily derived process to protect cells and tissues from a broad spectrum of insults. Unfortunately abrogating the insult comes at a cost with compromise of cell or tissue integrity. For the CNS to function it needs to rigorously control its microenvironment and thus is highly susceptible to negative sequelae of the IIR. In the course of studying neuroinflammation in a wide variety of human CNS diseases, we discovered an intriguing molecule, CHI3L1, that appears to be crucial in mitigating untoward CNS effects of IIR. During neuroinflammation the distribution of CHI3L1 is completely unlike that in other tissues. In systemic tissues CHI3L1 is expressed highly in macrophages during the IIR, however in the CNS macrophage expression appears to be severely down-regulated and instead astrocytes express high levels. Experimental studies in animal models of CNS disease have suggested that $\mathrm{CHI} 3 \mathrm{~L} 1$ is part of a unique anti-inflammatory pathway in the CNS. The mechanism of CHI3L1 down-regulation in CNS macrophages and the role of astrocytic up-regulation remains to be elucidated, however this novel pathway offers a new target to regulate $\mathrm{CNS}$ inflammation.

\section{Idiopathic Normal Pressure Hydrocephalus: Pathological changes in cortical biopsies in relation to function and response to treatment}

\author{
S. Sennik, M. Anton M.D., M. Cusimano M.D.,Ph.D., \\ D.G. Munoz, M.D.
}

St.Michael's Hospital and Keenan Research Centre of the Li Ka Shing Knowledge Institute, Toronto, Ontario, Canada

doi:10.1017/cjn.2015.261

Cortical peri-operative biopsies obtained from 11 idiopathic normal pressure hydrocephalus (iNPH) consenting patients (aged 67-88 years, median 74) were immuno-stained for Beta-amyloid (AMYB), Amyloid Precursor Protein (APP), total Tau, phosphorylated tau (AT8), Alpha Synuclein (AS), TDP- 43, P62 \& GFAP. 5/11 patients had AMYB deposits, 2 diffuse plaques only, 3 diffuse and cored plaques; 1 also with amyloid angiopathy (CAA). Plaque neurites were labeled for APP and AT8 in all 3 patients with cored plaques; in one were these and neurofibrillary tangles labeled for total tau. The surface area covered by AMYB ranged from $12.8 \%$ to $0.08 \%$. The presence of AMYB correlated with age $(\mathrm{t}, \mathrm{p}=.02)$. Neither AS nor TDP-43 pathologies were identified. The density of cortical GFAP + astrocytes showed a trend towards inverse correlation with age $(r=-0.58$, $\mathrm{p}=.06$ ) but not with AMYB or AT8 pathologies. There were no significant correlations between pre-shunt Evans Ratio and MoCA scores and presence or amount of pathology. Improvement in ventriculomegaly $(5 / 11)$ did not differ between patients with and without AMYB pathologies. Improvements in MoCA (6/8) were more common in patients without AMYB deposits $\left(X^{2}, \mathrm{p}=.028 ; \mathrm{t}, \mathrm{p}=.04\right)$, but also in younger patients $(\mathrm{t}, \mathrm{p}=.019)$. The patient with CAA improved, but the one with total tau pathology did not improve cognitively, although her gait did. In this preliminary study only AD-related pathology was found in iNPH. Cognitive improvement was associated with younger age and absence of amyloid deposits; however, a larger number of patients will be required to dissect these variables. 